\title{
Acogimiento en Familia Ajena y Visitas de los Menores con sus Padres Biológicos
}

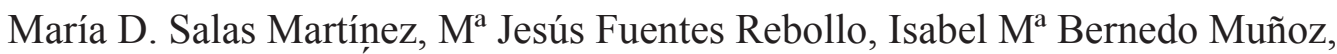 \\ Miguel Ángel García Martín y Sandra Camacho Pérez
}

Universidad de Málaga

Disponible online 30 de abril de 2009

\begin{abstract}
El acogimiento en familia ajena es una medida de protección escasamente estudiada en nuestro país. El objetivo de este estudio fue analizar la situación de los niños en acogimiento con familia ajena en la provincia de Málaga y evaluar la calidad de las visitas de éstos con sus padres biológicos. Los participantes fueron 95 menores, 72 familias biológicas y 69 familias acogedoras. El instrumento utilizado para la obtención de información fue la Ficha de Recogida de Datos diseñada para el estudio. El trabajo se realizó con la participación del Servicio de Protección de Menores y de las ICIFs de la provincia de Málaga. Los resultados muestran: a) presencia de maltrato en todos los niños y niñas, habiendo estado la mayoría de ellos acogimiento residencial previo; b) alto porcentaje de padres y madres biológicos con problemas de toxicomanía; c) bajo porcentaje de acogimientos remunerados; d) pocos contactos entre familias acogedoras y biológicas; e) bajo cumplimiento del régimen de visitas establecido; y f) evaluación negativa de las visitas por parte de los técnicos.
\end{abstract}

Palabras clave:

Acogimiento en familia ajena, acogedores, familia biológica, visitas de los padres.

Family foster care is a measure of protection little studied in our country. The aim of this research is to analyse the situation of children in foster care and to evaluate the quality of the visits between children with biological parents. The participants were 95 children, 72 biological families and 69 foster families. The instrument used was the "Child File Summary Form" which was designed for the study. Access to the information about the care situations was done through the Child and Family Protection Service and the associations which supervise foster care in Malaga. The results show: a) the presence of maltreatment in all the children and previous residential care in the majority of children; b) great incidence of biological parents with problems of substance abuse; c) a low percentage of foster care is remunerated and few contacts are observed between foster and biological parents; d) parental visits are infrequent and sporadic; e) many contacts are evaluated negatively by the technical staff.

Keywords:

Foster care, foster parents, foster families, parent visitation.

\footnotetext{
Este estudio forma parte de un Proyecto de Investigación de Excelencia financiado por la Consejería de Innovación, Ciencia y Empresa de la Junta de Andalucía (Dirección General de Investigación, Tecnología y Empresa) (P07-SEJ-02647).

Correspondencia: Dña. María D. Salas Martínez. Dpto. Psicología Evolutiva y de la Educación. Facultad de Psicología. Campus de Teatinos s/n. Universidad de Málaga. Teléfono: (+34) 9521375 18. Fax : (+34) 9521324 83. E-mail: m.salas@uma.es
} 
El acogimiento familiar es considerado de forma unánime, como el emplazamiento más aconsejable para los niños y niñas que han tenido que ser separados de sus familias (Del Valle, López, Montserrat y Bravo, 2008). El objetivo de este tipo de medida es que el niño o niña conviva y se integre en una familia acogedora, evitando su estancia en una institución. La duración y características de cada acogimiento podrán variar en función de las necesidades y circunstancias de cada niño. Según la duración, los acogimientos pueden ser simples, con una temporalidad máxima de dos años y permanentes, sin limitación temporal. Tanto unos como otros se pueden constituir en familia extensa (cuando el acogimiento se lleva a cabo por familiares del menor) o en familia ajena (cuando se realiza por una familia con la que el niño no tiene lazos de parentesco).

Un niño o niña es incluido en un programa de acogimiento simple cuando se produce una situación de crisis en la familia de origen que desencadena la separación, pero se prevé que ésta se recupere y, por tanto, se pueda producir la reunificación familiar. También se utiliza este tipo de acogimiento durante un periodo determinado de tiempo, mientras se busca una medida más estable. El acogimiento familiar permanente se realiza cuando no existe previsión de retorno del niño con su familia de origen y además las características de la situación hacen conveniente la integración estable y duradera en otra familia.

El acogimiento familiar en el Sistema de Protección de la Infancia en España requiere un análisis profundo de todos los factores que entran en juego, así como, del grado de cumplimiento de los objetivos para los que se han planteado este tipo de recursos, ya que hasta el momento las investigaciones realizadas son escasas e incompletas y no permiten llegar a conclusiones claras sobre su funcionamiento, el establecimiento de medidas y la elaboración de programas y herramientas para conseguir la mejora de éste.

Hasta el momento, las investigaciones realizadas en nuestro país han sido, fundamentalmente, estudios centrados en acogimientos en familia extensa o trabajos que engloban las distintas modalidades de acogimiento. Por ejemplo, los trabajos de Del Valle, Álvarez-Baz y Bravo (2002), realizado en el Principado de Asturias, de Montserrat $(2006,2007)$ en Barcelona y de Molero, Moral, Albiñana, Sabater y Sospedra, (2007) en Valencia, son investigaciones específicas sobre acogimiento en familia extensa, que comprenden estudios descriptivos de la situación de estos acogimientos. En Andalucía también se han realizado algunas investigaciones centradas en la situación del acogimiento en familia extensa (Bernedo, 2004; Lumbreras, 2003; Lumbreras, Fuentes, y Bernedo, 2005).

Un trabajo que es punto de referencia y que incluye otras modalidades de acogimiento, es el estudio llevado a cabo por Amorós, Palacios, Fuentes, León y Mesas (2003) sobre la evaluación del Programa de Familias Canguro, en el que se estudiaron longitudinalmente los diferentes tipos de acogimientos familiares en nueve Comunidades Autónomas. Balluerka, Gorostiaga, Herce y Rivero (2002), también realizaron un estu- dio en la Comunidad Autónoma del País Vasco que englobaba tanto acogimiento en familia extensa como ajena.

Otra investigación reciente que abarca estos dos tipos de acogimiento, fue la llevada a cabo por Palacios y Jiménez (2007) en Andalucía. En este trabajo se realizó un muestreo aleatorio de las familias acogedoras en las ocho provincias andaluzas. La muestra estuvo compuesta por un total de 218 menores y sus correspondientes familias acogedoras. Una de las conclusiones más relevantes de este estudio fue la baja presencia de contactos y visitas entre los progenitores y sus hijos. En los acogimientos con familia ajena se obtuvo que más del $70 \%$ de los niños no recibían visitas de los padres, y alrededor del $45 \%$, no tenían contactos con la madre. Además, aproximadamente el $30 \%$ de los acogedores de familia ajena hicieron una valoración negativa del impacto de las visitas sobre los niños.

También algunos trabajos nacionales tuvieron en cuenta el estudio de los contactos de los niños con sus familias. Así por ejemplo, el trabajo realizado por Balluerka et al. (2002), también estudió las visitas de los menores con sus familias de origen, obteniéndose que los menores con pocas visitas y/o visitas supervisadas mostraron mejor autoconcepto que aquellos que tenían más visitas o éstas no eran supervisadas.

Otro estudio en nuestro país (León y Palacios, 2004) analizó la frecuencia de las visitas durante el acogimiento, en relación con la medida de protección adoptada al final del acogimiento, en una muestra de 120 niños y niñas acogidos. Se halló que la frecuencia de las visitas se asociaba positivamente al regreso de los niños con sus padres. Así mismo, se encontró asociación entre la detección de situaciones conflictivas entre padres e hijos durante las visitas y el fracaso de la reunificación.

Estudios en otros países han mostrado evidencias de que los niños en acogimiento con familia ajena mantienen menor continuidad en el contacto con sus padres biológicos (Berrick, Barth y Needell, 1994; Pecora, Le Prohn y Nasuti, 1999). Este hecho repercute de mayor modo en el caso de los acogimientos simples, si tenemos en cuenta que muchos estudios han mostrado que para aquellos niños que reciben visitas de sus padres es mucho más probable la reunificación (Cleaver, 2000; Testa y Slack, 2002).

Los investigadores Benedict y White (1991) y Oyserman y Benbenishty (1992) llevan años argumentando que los contactos regulares de los padres con sus hijos, cuando éstos se hallan en acogimiento, contribuyen a la estabilidad, continuidad y fomento de los vínculos afectivos y de las relaciones entre padres e hijos, por lo que su preservación se ha convertido en una práctica importante en la protección de la infancia.

Algunos estudios aconsejan la realización de visitas a lo largo del período de acogimiento por sus efectos positivos en el ajuste del niño a su nuevo entorno, por la disminución de la probabilidad de fracaso del acogimiento y por la reducción del tiempo medio de estancia con la familia de acogida (Benedict y White, 1991). Trabajos como los de Haight, Black, Workman y Tata (2001), Leathers (2003) y McWey y Mullis (2004) revelan 
que la implicación de los padres biológicos y la frecuencia de las visitas poseen un efecto indirecto sobre la adaptación de los niños a la familia de acogida, y directo sobre los vínculos de apego con los familiares.

Como se ha visto a lo largo de esta revisión, es necesario continuar analizando la situación del acogimiento en familia ajena. Además, partiendo de los datos hallados en el trabajo de Palacios y Jiménez (2007) en cuanto al bajo porcentaje de visitas existentes y al beneficio que estos contactos tienen para la consecución de los objetivos del acogimiento (Haight et al., 2001; Leathers, 2003; León y Palacios, 2004; McWey y Mullis, 2004), resulta indispensable estudiar con profundidad el funcionamiento de las visitas.

El objetivo principal de este estudio es analizar los acogimientos con familia ajena de la provincia de Málaga y el estado actual de las visitas que mantienen los menores con sus padres biológicos. En concreto se pretende conocer:

a) Las características de los niños y niñas que están en acogimiento familiar,

b) las características de las familias biológicas y de las circunstancias que provocaron la separación de éstas y sus hijos,

c) las características de las familias acogedoras,

d) los tipos de acogimientos (simples / permanentes) más frecuentes en nuestra provincia, las diferencias entre ellos y el cumplimiento de las medidas adoptadas,

e) la relación entre los niños y niñas con sus padres biológicos mediante la información proporcionada por los informes de seguimiento y por los técnicos sobre las visitas y contactos entre ellos.

Método

\section{Participantes}

Los datos de esta investigación describen todos los acogimientos con familia ajena vigentes en la provincia de Málaga hasta abril de 2007. La muestra se compone de 95 sujetos (55 niños y 40 niñas) con una media de edad de 8 años, con edades comprendidas entre 1 y 18 años. Los padres biológicos de los niños y niñas de este estudio forman 72 familias (57 padres y 69 madres). Los padres tienen una media de edad de 43,8 años, y las madres de 36,2 años. Por último, 69 familias acogedoras participan en el estudio, siendo la edad media de los acogedores 45,3 años y la de las acogedoras 44 años.

\section{Instrumentos}

Se utilizó una Ficha de Recogida de Datos diseñada para recopilar la información de los expedientes de acogimiento que figuran en el Servicio de Protección de Menores de la Delegación Provincial de Málaga y en las ICIFs.
En esta ficha se recogen datos sobre los niños y niñas en acogimiento (edad, etnia, motivos del desamparo, tipo de maltrato sufrido, acogimientos previos, etc.), las familias biológicas (edad, estudios, ocupación, problemática que presentan, etc.), las familias acogedoras (edad, estudios, ocupación, hijos biológicos, acogimientos previos, etc.), y la calidad de las interacciones de los padres y madres con sus hijos durante las visitas valoradas por los técnicos.

\section{Procedimiento}

Este estudio se ha realizado con la participación del Servicio de Protección de Menores, las ICIFs que supervisan los acogimientos en la provincia de Málaga y el grupo de investigación sobre acogimiento y adopción de la Universidad de Málaga. El procedimiento de trabajo consistió en la visita por parte de miembros del grupo de investigación a las sedes de la Delegación del Servicio de Protección y de las ICIFs, donde se tuvo acceso a los expedientes de los niños y de las familias, así como a los informes de seguimiento realizados. La recogida de datos se efectuó desde Abril de 2006 hasta Abril de 2007.

Las interacciones de los padres y madres con sus hijos durante las visitas fueron evaluadas por los técnicos que se encuentran presentes durante dichas visitas, en función de la calidad en la interacción que se observaba entre ellos. El criterio de valoración fue el siguiente: a) interacción positiva cuando los padres se implicaban muy activamente en la interacción con sus hijos, les hablaban y jugaban con ellos; b) interacción media cuando los padres no se implicaban siempre en la interacción y en ocasiones permanecían pasivos; c) interacción negativa cuando los padres permanecían pasivos en numerosas ocasiones, sin implicarse claramente en la interacción con sus hijos, sin hablar y sin jugar con ellos o cuando el tipo de interacción que tenían era claramente negativa (mensajes inadecuados, evidencias de malestar en el niño, etc.).

\section{Resultados} a) Características de los acogimientos y de los niños y niñas
acogidos

De los 95 niños y niñas que en Abril de 2007 estaban en acogimiento con familia ajena en la provincia de Málaga, 67 estaban en acogimiento permanente (70,5\%), y 28 niños en simple (29,5\%). De ellos, 35 niños estaban acogidos con hermanos, de los cuales, 32 estaban con un hermano y 3 con dos hermanos.

La edad media de los niños en acogimiento en el momento de la recogida de los datos era de 8 años $(D T=4,34)$, con un rango entre 1 y 18 años. En cuanto a la etnia, 53 acogidos eran de la misma etnia que sus acogedores y 42 de etnia diferente (gitanos y marroquíes). Respecto a la discapacidad, 82 menores 
no tenían discapacidad, 6 tenían una discapacidad grave (parálisis cerebral e hidrocefalia) y 7 leve (problemas auditivos y motóricos).

Según los datos de los expedientes, todos los niños han sido víctimas de algún tipo de maltrato por parte de sus progenitores. Se diferenció entre maltrato activo (maltrato físico, maltrato emocional o psíquico y abuso sexual) y maltrato pasivo (negligencia o abandono físico, negligencia o abandono emocional o psíquico) (López, López, Fuertes, Sánchez y Merino, 1995). Según esta clasificación, el 67,4\% de los menores sufrió maltrato pasivo y el $32,7 \%$ maltrato activo, de los que el 5,3\% sufrió abuso sexual. Es importante aclarar que se ha señalado el tipo de maltrato más grave sufrido por el menor, aunque en numerosas ocasiones, cuando ha habido un maltrato activo, también lo ha habido pasivo.

Los motivos por los que se produjo el desamparo de los niños y niñas fueron, en el 38,9\% de los casos la drogadicción, en el 26,3\% el abandono, en el 25,3\% la psicopatología, y en el $9 \%$ la estancia en prisión de uno o ambos progenitores. En los casos en los que la problemática era múltiple se recogió el motivo más directamente relacionado con el desamparo.

El 93,7\% de los niños y niñas que forman parte del estudio estuvieron en acogimiento residencial antes de iniciar el acogimiento familiar, la mayoría de los cuales $(76,9 \%)$ estuvieron en un solo centro, y el 16,8\% en dos centros. Sólo 6 menores no estuvieron en centros antes de iniciar el acogimiento (Tabla 1).

Tabla 1. Duración del acogimiento residencial.

\begin{tabular}{lcc}
\hline & $n$ & Porcentaje \\
\hline No acogimiento residencial & 6 & 6,3 \\
Hasta 6 meses & 27 & 28,4 \\
De 6 meses a 1 año & 32 & 33,7 \\
De 1 año a 2 años & 14 & 14,7 \\
De 2 a 3 años & 12 & 12,6 \\
Más de 3 años & 4 & 4,2 \\
Total & 95 & 100 \\
\hline
\end{tabular}

El $30,5 \%$ de los niños y niñas tuvieron un acogimiento familiar previo al actual, de los cuales, el 8,4\% fue con familia extensa y el $22,1 \%$ con familia ajena. El 78,6\% estuvieron menos de 1 año en acogimiento familiar previo, ya sea con familia ajena o extensa.

En cuanto a la duración del acogimiento actual, se observa que el $71 \%$ de los acogimientos permanentes y el $25 \%$ de los simples llevan constituidos más de dos años.

Por último, en cuanto a los cambios en el tipo de medida de protección se observa que el $29,5 \%$ de los niños y niñas que se encontraban en acogimiento simple han pasado a acogimiento permanente, y de ellos, el 27,4\% siguen estando con la misma familia, mientras que el $2,1 \%$ ha pasado a estar acogido con una familia diferente.

\section{b) Características de la familia biológica}

Entre las características de las familias biológicas se ha encontrado que el 19,3\% de los padres y el 36,1\% de las madres presentan psicopatología (en el $22,6 \%$ y el $4,2 \%$ respectivamente, no hay datos). El 27,8\% de los padres y el 26,4\% de las madres se encuentran en prisión. El $44,4 \%$ de los padres y el $45,8 \%$ de las madres son toxicómanos, recibiendo en la actualidad tratamiento solamente el 4,2\% de los padres y el 11,1 de las madres. Varios casos de padres y madres presentan dos o más problemáticas.

Respecto al nivel de estudios se tienen datos de 13 padres y 19 madres. 9 padres no poseen estudios, 3 tienen estudios primarios y 1 universitarios. De las madres, 10 no poseen estudios, 7 tienen estudios primarios y 2 secundarios.

En relación a la ocupación de los padres, 10 se encuentran en paro, 23 trabajan ocasionalmente, 7 tienen trabajo estable y 6 son pensionistas. De las madres, 37 se encuentran en paro, 20 realizan trabajos ocasionales, 5 tienen trabajo estable y 4 son pensionistas. De 11 padres y 3 madres no existen datos al respecto.

\section{c) Características de las familias acogedoras}

De las 69 familias acogedoras que constituyen la muestra, 59 son parejas heterosexuales, 2 homosexuales, y 8 monoparentales. La composición de los hogares de acogida (incluyendo a los acogidos) es la siguiente: el 4,3\% está formado por dos miembros, el $14,5 \%$ por tres, el $26,1 \%$ por cuatro, el $31,9 \%$ por cinco, el $15,9 \%$ por seis, y el $7,1 \%$ por más de seis miembros. En cuanto a hijos biológicos, el $23,2 \%$ de las familias acogedoras no tiene hijos, el 17,4\% tiene un hijo, el 37,7\% dos, el 18,8\% tres, y el $2,9 \%$ cuatro. De las 69 familias, 49 tienen un solo niño acogido $(55,8 \%), 16$ familias tienen a dos $(23,2 \%)$ y 4 a tres $(5,8 \%)$.

Respecto al nivel de estudios, el 30\% de los acogedores posee estudios primarios, el 31,7\% secundarios o FP, y el 38,3\% estudios universitarios. De las acogedoras, el 1,4\% no posee estudios, el 27,5\% tiene estudios primarios, el 33,3\% secundarios y $\mathrm{FP}$, y el $37,7 \%$ universitarios.

En cuanto a la ocupación de los acogedores, el 95\% tiene un trabajo estable, y el 5\% son pensionistas, no estando ninguno de ellos en situación de paro. En el caso de las acogedoras $13 \%$ de ellas están en paro, el 8,7\% trabaja ocasionalmente, el $73,9 \%$ de forma estable, y el 4,3\% es pensionista.

Referente a la remuneración, el 78,9\% de los acogimientos no están remunerados y el $21,1 \%$ reciben una cantidad mensual de entre 200 y 300 euros por menor acogido, recibiendo estas cantidades los acogimientos de grupos de hermanos o los casos de niños o niñas que tienen discapacidad.

Respecto a los acogimientos previos, el $66,7 \%$ de las familias no ha realizado ningún acogimiento anterior al actual, mientras que el 20,3\% tuvo un acogimiento previo, el 5,8\% dos, el 1,4\% tres, y el 5,7\% más de tres. 
Por último, en cuanto a la relación entre las familias biológicas y las de acogida, en el $63,8 \%$ de los casos no existe ningún tipo de contacto, mientras que en el $36,2 \%$ existe relación. Los técnicos de las ICIFs que realizan los seguimientos valoran estas relaciones como buenas en el 33,3\% de los casos, y como malas en el $2,9 \%$.

\section{b) Visitas de los niños y niñas con sus familiares}

De los 95 menores que formaban la muestra, 70 recibieron visitas sus padres o de alguno de ellos, mientras que 25 no. En la Tabla 2 pueden observarse los niños que tuvieron visitas de sus progenitores según el tipo de acogimiento (simple o permanente).

Tabla 2. Visitas o contactos de los padres según tipo de acogimiento.

\begin{tabular}{|c|c|c|c|c|}
\hline & \multicolumn{2}{|c|}{$\begin{array}{c}\text { Visitas de progenitores en } \\
\text { simples }\end{array}$} & \multicolumn{2}{|c|}{$\begin{array}{c}\text { Visitas de progenitores en } \\
\text { permanentes }\end{array}$} \\
\hline & $n$ & Porcentaje & $n$ & Porcentaje \\
\hline Sí & 21 & 75 & 49 & 73,1 \\
\hline No & 7 & 25 & 18 & 26,9 \\
\hline Total & 28 & 100 & 67 & 100 \\
\hline
\end{tabular}

Respecto al número de niños que recibieron visitas específicamente de sus padres, sólo 35 de los 95 que componen la muestra tuvieron algún encuentro con ellos $(36,8 \%)$, mientras que 63 de ellos tuvieron visitas de las madres $(66,3 \%)$.

Teniendo en cuenta el total de visitas posibles que cada menor tiene establecidas (trimestral, mensual, quincenal, semanalmente, etc.) a lo largo de un año y el número de visitas a las que realmente han acudido los padres en ese tiempo, se ha hallado la regularidad con la que estos niños recibieron visitas de sus padres y madres. Esta regularidad se ha categorizado como todas las posibles, $3 / 4$ partes, $2 / 4$ partes, $1 / 4$ parte y menos de 1/4 de las establecidas en el régimen de visitas (Tabla 3).

Tabla 3. Regularidad de las visitas de los padres y madres.

\begin{tabular}{lcccc}
\hline & \multicolumn{2}{c}{ Padres } & \multicolumn{2}{c}{ Madres } \\
\hline & $n$ & Porcentaje & $n$ & Porcentaje \\
\hline Todas las posibles & 9 & 25,7 & 19 & 30,2 \\
3/4 de las posibles & 9 & 25,7 & 7 & 11,1 \\
2/4 de las posibles & 10 & 28,6 & 21 & 33,3 \\
1/4 de las posibles & 3 & 8,6 & 9 & 14,3 \\
$<1 / 4$ posibles & 4 & 11,4 & 7 & 11,1 \\
Total & 35 & 100 & 63 & 100 \\
\hline
\end{tabular}

Se ha estudiado la relación entre la regularidad del cumplimiento del régimen de visitas y el motivo del desamparo. Para ello, se han realizado análisis de variables categóricas mediante tablas de contingencia y para conocer el grado de relación entre éstas se ha empleado el estadístico Chi-Cuadrado $\left(\chi^{2}\right)$. Se han encontrado diferencias estadísticamente significativas, en el caso de las madres, $\chi^{2}(12, N=63)=23,83, p<, 05$ (Tabla 4). Las madres que cumplen con mayor frecuencia el régimen de visitas establecido son las que fueron separadas de sus hijos por abandono $(47,1 \%)$. Cuando las madres presentan problemas de toxicomanía suelen acudir solamente a la mitad de las vistas establecidas $(57,1 \%)$. No se han encontrado diferencias significativas en la relación de estas dos variables en el caso de los padres.

Tabla 4. Tabla de contingencia entre el motivo del desamparo y la regularidad de las visitas de las madres.

\begin{tabular}{lccccccc}
\hline \multicolumn{7}{c}{ Regularidad de las visitas de la madre (\%) } \\
\hline & $\begin{array}{c}\text { Todas las } \\
\text { posibles }\end{array}$ & $\begin{array}{c}3 / 4 \text { de las } \\
\text { posibles }\end{array}$ & $\begin{array}{c}1 / 2 \text { de las } \\
\text { posibles }\end{array}$ & $\begin{array}{c}1 / 4 \text { de las } \\
\text { posibles }\end{array}$ & $\begin{array}{c}<1 / 4 \\
\text { posibles }\end{array}$ & $\chi^{2}$ & $g l$ \\
\hline Drogadicción & 19 & 0 & 57,1 & 14,3 & 9,5 & & \\
Psicopatología & 31,3 & 12,5 & 18,8 & 18,8 & 18,8 & & \\
Prisión & 22,2 & 44,4 & 33,3 & 0 & 0 & & \\
Abandono & 47,1 & 5,9 & 17,6 & 17,6 & 11,8 & & \\
Total & 30,2 & 11,1 & 33,3 & 14,3 & 11,1 & $23,83 *$ & 12 \\
\hline$p<05$ & 78 & & & &
\end{tabular}

\section{Interacciones con el padre y la madre durante las visitas}

En el caso de los padres, se ha valorado que del $38,6 \%$ que asisten a las visitas, el 37,1\% presenta un grado de interacción positivo, ya que se implican muy activamente en la interacción con sus hijos. E1 34,3\% presenta un nivel medio de interacción y el $28,6 \%$ presenta una interacción negativa. Respecto a las madres que acuden a las visitas (66,3\%), la interacción con sus hijos se ha valorado como positiva en el $33,3 \%$, media en el $27 \%$, y negativa en el $39,7 \%$.

Por otro lado, se ha examinado la relación entre la valoración realizada sobre la calidad en esta interacción y el motivo del desamparo por el que los padres fueron separados de sus hijos (toxicomanía, prisión, psicopatología y abandono). El análisis realizado mediante el Chi-Cuadrado $\left(\chi^{2}\right)$ pone de manifiesto la existencia de diferencias estadísticamente significativas entre las frecuencias de las valoraciones del tipo de interacción presentadas y las frecuencias de los motivos de desamparo en el caso de las madres, $\chi^{2}(6, N=63)=14,03, p<, 05$. En el caso de presencia de psicopatología en las madres la interacción es más negativa $(68,8 \%)$ que en los otros motivos de desamparo. Por el contrario, en los casos en los que el motivo de desamparo fue drogadicción o abandono se observan interacciones más positivas, $47,6 \%$ y $47,1 \%$, respectivamente (Tabla 5).

Tabla 5. Tabla de contingencia entre las variables motivo del desamparo y calidad en la interacción de los niños con sus madres durante las visitas.

\begin{tabular}{|c|c|c|c|c|c|}
\hline & \multicolumn{3}{|c|}{ Interacción entre madre e hijo/a } & \multirow[b]{2}{*}{$\chi^{2}$} & \multirow[b]{2}{*}{$g l$} \\
\hline & Positiva (\%) & Media (\%) & Negativa (\%) & & \\
\hline Drogadicción & 47,6 & 19 & 33,3 & & \\
\hline Psicopatología & 0 & 31,5 & 68,8 & & \\
\hline Prisión & 33,3 & 44,4 & 22,2 & & \\
\hline Abandono & 47,1 & 23,5 & 29,4 & & \\
\hline Total & 33,3 & 27 & 39,7 & $14,03 *$ & 6 \\
\hline
\end{tabular}

En el caso de los padres, aunque no se han encontrado diferencias estadísticamente significativas entre las valoraciones 
del tipo de interacción presentadas y los motivos de desamparo, también se encontró el mayor número de interacciones calificadas como negativas, cuando el padre presentaba psicopatología $(62,5 \%)$.

\section{Discusión}

Los resultados obtenidos en este estudio permiten conocer las características del perfil de los protagonistas directos del acogimiento con familia ajena (niños/as, familias biológicas y familias acogedoras), así como evaluar la relación de los menores con sus padres durante las visitas entre ellos.

Características de los acogimientos y de los niños y niñas acogidos

De los 95 niños que forman la muestra, 35 están acogidos con hermanos. Los estudios sobre grupos de hermanos en acogimiento, muestran resultados positivos cuando los hermanos son acogidos juntos (Smith, 1996, 1998). Además, se ha visto que los acogidos en familia ajena muestran un riesgo especialmente alto de ser separados de sus hermanos (Shlonsky, Webster y Needell, 2003), por lo que el alto número de niños que se encuentran acogidos con sus hermanos, muestra que la política seguida por los técnicos a la hora de constituir los acogimientos tiene en cuenta esta variable tan importante para el bienestar de los menores.

Todos los niños que formaron parte del estudio sufrieron maltrato, siendo maltrato pasivo en el $67,4 \%$ de los casos y el resto maltrato activo. En otros estudios (Amorós et al., 2003, Palacios y Jiménez, 2007) se han encontrado porcentajes más bajos. La diferencia puede deberse a que las experiencias de maltrato son significativamente más frecuentes en los acogidos en familia ajena que en extensa, y en nuestro estudio todos acogimientos son en familia ajena.

Un alto porcentaje $(93,7 \%)$ de niños estuvieron en acogimiento residencial antes de iniciar el acogimiento familiar. También en el estudio de Palacios y Jiménez (2007) se obtuvo que el $91 \%$ de los acogidos con familia ajena tuvieron acogimientos previos, aunque en este caso no se diferenció entre acogimientos con otras familias o residencial. Esto muestra que los niños siguen pasando largos períodos de institucionalización antes de llegar a una familia, por lo que se debe seguir trabajando en potenciar el acogimiento familiar como principal medida de protección.

Por otro lado, se encontró que un $25 \%$ de acogimientos simples tenía más de 2 años de duración, cuando éste es el tiempo máximo previsto para este tipo de medida. Lo que define principalmente al acogimiento simple es su carácter provisional, en espera de la posible reintegración del menor con su familia biológica o de la búsqueda de otra medida más estable para el niño o niña. Por ello, estos acogimientos simples de larga duración pueden estar indicando la necesidad de que los seguimientos se realicen con una mayor frecuencia.

\section{Características de las familias biológicas}

El análisis de la problemática que presentaban los padres cuando fueron separados de sus hijos y que supuso el motivo del desamparo, muestra que la toxicomanía era el problema más común, tanto en los padres $(44,4 \%)$, como en las madres $(45,8 \%)$ de los menores acogidos. Los estudios revisados (Amorós et al., 2003; Bernedo, 2004; Del Valle et al., 2002; Gibbs y Múller, 2000; Lumbreras, 2003; Palacios y Jiménez, 2007) coinciden con estos resultados respecto a la alta frecuencia de toxicomanía en los padres y madres de los niños acogidos. Además, sólo el 4,2\% de los padres y el 11,1\% de las madres se encontraban en programas de rehabilitación en el momento de la recogida de datos. De este modo, se confirma que el problema de la drogadicción sigue siendo una de las principales causas de desestructuración familiar y la necesidad de que esta población participe en mayor medida en programas de rehabilitación.

En cuanto al nivel educativo de los progenitores de los menores, los datos que aparecen en los expedientes son muy escasos. Así, en el 77,2\% de los padres y el 72,5\% de las madres, no figura este dato en los expedientes. Del resto, la mayoría no tenía estudios, tanto en el caso de los padres como de las madres. En cuanto a la situación laboral, el 58\% de los padres y el $83 \%$ de las madres estaban en paro o tenían trabajos ocasionales, lo que sigue poniendo de relieve la problemática social que este colectivo presenta.

\section{Características de las familias acogedoras}

Los datos sociodemográficos obtenidos sobre las familias acogedoras son muy similares a los de otras investigaciones, tanto respecto a la edad media de acogedores y acogedoras, como al nivel educativo (alto porcentaje de acogedores y acogedoras con estudios universitarios) y situación laboral de éstos (muy pocas situaciones de paro). Estos datos dan cuenta, de que el sector de la población que está ejerciendo la labor de acogimiento son familias de un nivel sociocultural medio-alto.

En relación a la estructura de las familias acogedoras, se ha encontrado que presentan un perfil muy tradicional, siendo la gran mayoría parejas heterosexuales, con muy pocas situaciones de familias monoparentales o de parejas homosexuales, lo que sigue coincidiendo con lo encontrado en otros estudios. Del Valle et al. (2008) sugieren que incorporar otras situaciones distintas a las formas clásicas de estructura familiar podría ser una buena manera de ampliar el conjunto de familias acogedoras disponible. Sería interesante averiguar si el bajo número de parejas homosexuales o de personas solas que acogen, es debido a que las campañas de difusión no están dirigidas a estos 
sectores de la población, a un bajo interés de éstos o a criterios de las valoraciones de idoneidad.

También resulta interesante el hecho de que la mayoría de los acogedores tienen hijos biológicos $(76,8 \%)$, lo que podría ser debido a que en las campañas de difusión del acogimiento familiar, se señala que la principal motivación para ser acogedor es la ayuda a un niño o niña que ha sido separado de su familia y no el tener la experiencia de paternidad o maternidad, diferenciando claramente esta medida de protección de la infancia de la adopción.

Respecto a la remuneración, los datos obtenidos dan cuenta de los pocos acogimientos de familia ajena que están recibiendo ayuda económica en nuestra provincia, quedando ésta reducida únicamente a aquellos casos de acogimientos de dos o más hermanos o de niños con algún tipo de discapacidad. Otras investigaciones a nivel nacional han señalado las escasas remuneraciones como una de las mayores quejas de las familias acogedoras (Del Valle et al., 2002; Del Valle et al., 2008; Palacios y Jiménez, 2007), por lo que, un mayor apoyo material a estas familias desde las administraciones contribuiría a que existiesen más familias dispuestas a acoger y facilitaría el trabajo de las que ya lo hacen.

Un dato muy positivo que hemos obtenido en los resultados, es el alto porcentaje de familias que han tenido acogimientos previos al actual (43,3\% de las familias participantes). Ello parece dar cuenta de que la experiencia que han tenido las familias acogedoras ha sido positiva, y que, a pesar de algunas de las cuestiones claramente mejorables, el trabajo y esfuerzo de la Administración y los técnicos de las ICIFs para impulsar el acogimiento familiar como medida de protección, está dando sus frutos.

Por último, existe un bajo porcentaje de familias acogedoras que tengan contactos con las familias biológicas, pero cuando éstos existen son valorados positivamente en su gran mayoría. Otros estudios indican que la existencia de relaciones entre ambas familias (especialmente en los acogimientos simples) ha permitido el establecimiento de una mejor comunicación y entendimiento entre ellas, y en consecuencia con el niño o niña acogido (Del Valle et al., 2008). Creemos que es algo a seguir investigando, ya que, puede que los casos en los que se ha visto este efecto positivo sean aquellos en los que las familias biológicas estaban abiertas a la colaboración y no presentaban una problemática social extrema.

\section{Visitas}

Los porcentajes obtenidos sobre las visitas de los menores con sus padres son muy similares a los encontrados en otros estudios (Del Valle et al., 2002; Hunt, 2003; Palacios y Jiménez, 2007; Pecora, et al., 1999), siendo la regularidad en el cumplimiento del régimen establecido muy bajo y similar en padres y madres. El dato es aún más preocupante, cuando se analiza por separado la existencia de visitas de padres y madres en los casos de acogimientos simples. Así, sólo el 75\% de los niños en acogimiento simple recibe visitas de sus progenitores, existiendo 7 niños en este tipo de acogimiento que no tienen visitas de ninguno de sus padres. Este resultado requiere que se investigue con más profundidad qué ocurre con las visitas en los acogimientos simples, ya que el mantenimiento de los vínculos afectivos por medio de los encuentros familiares es un objetivo prioritario en esta modalidad de acogimiento (Amorós et al., 2003).

Al relacionar la regularidad del cumplimiento de las visitas con los motivos por los que estos niños fueron separados de sus progenitores, se encontró que en el caso de las madres, las que más cumplen el régimen de visitas establecido son aquellas que fueron separadas de sus hijos por abandono. Esto podría deberse a que estas madres no presenten una problemática tan grave como, por ejemplo, en el caso de la toxicomanía, y por ello, durante el acogimiento se observa que la regularidad en el contacto con sus hijos es mayor que en otros motivos de desamparo.

En cuanto a la calidad de las interacciones, la alta incidencia de interacciones calificadas como negativas muestra nuevamente datos poco satisfactorios sobre las visitas. Lo cual podría estar indicando, la necesidad de una mejor preparación de las visitas, sobre todo en el caso de las madres que presentan psicopatología.

Por último, sería conveniente realizar investigaciones longitudinales que recogiesen los distintos cambios e itinerarios por los que pasan los niños y niñas acogidos, ya que el acogimiento familiar es una medida proclive a los cambios (reinserciones con la familia biológica, cambios en el tipo de medida o suspensiones de las visitas, etc.).

\section{Referencias}

Amorós, P., Palacios, J., Fuentes, N., León, E. y Mesas, A. (2003). Familias Canguro. Una experiencia de protección a la infancia. Barcelona: Fundació "La Caixa".

Balluerka, N., Gorostiaga, A., Herce, C. y Rivero, A.M. (2002). Elaboración de un inventario para medir el nivel de integración del menor acogido en su familia acogedora. Psicothema, 14(3), 564-571.

Benedict, M. y White, R. (1991). Factors associated with foster care length of stay. Child Welfare, 70, 45-58.

Bernedo, I. M. (2004). Adolescentes acogidos por sus abuelos: Relaciones familiares y problemas de conducta. Tesis doctoral. Publicado por el servicio de Publicaciones de la Universidad de Málaga (SPICUM). Málaga: Universidad de Málaga.

Berrick, J. D., Barth, R. P. y Needell, B. (1994). A comparison of kinship foster homes and foster family homes: Implications for kinship foster care as family preservation. Children and Youth Services Review, 16, 33-63. 
Cleaver, H. (2000). Fostering Family Contact. London: The Stationery Office.

Del Valle, J. F., Álvarez-Baz, E. y Bravo, A. (2002). Acogimiento en familia extensa. Perfil descriptivo y evaluación de necesidades en una muestra del Principado de Asturias. Bienestar y Protección Infantil, 1(1), 34-56.

Del Valle, J. F., López, M., Montserrat, C. y Bravo, A. (2008). El acogimiento familiar en España. Una evaluación de resultados. Madrid: Ministerio de Educación, política social y deporte.

Gibbs, P. y Müller, U. (2000). Kinship foster care. Moving to the mainstream controversy, policy and outcomes. Adoption Quarterly, 4(2), 57-87.

Haight, W., Black, J., Workman, C. y Tata, L. (2001). Parentchild interaction during foster care visits: Implications for practice. Social Work, 46, 325-338.

Hunt, J. (2003). Family and Friends Carers. Scoping paper prepared for the Department of Health. London: DoH. Recuperado el 15 de septiembre de 2008 de: www. doh. gov.uk/carers/familyandfriends.

Leathers, S. J. (2003). Parental visiting, conflicting allegiances, and emotional and behavioral problems among foster children. Family Relations, 52, 53-63.

León, E. y Palacios, J. (2004). Las visitas de los padres y la reunificación familiar tras el acogimiento. Portualia, 4, 241-248.

López, F., López, B., Fuertes, J., Sánchez, J. M. y Merino, J. (1995). Necesidades de la infancia y protección infantil. Actuaciones frente a los malos tratos y desamparo de menores. Madrid: Ministerio de Asuntos Sociales.

Lumbreras, H. (2003). Perfil descriptivo de los acogimientos con familia extensa existentes en la provincia de Málaga. Trabajo de suficiencia investigadora. Universidad de Málaga. Documento sin publicar.

Lumbreras, H., Fuentes, M. J. y Bernedo, I. M. (2005). Perfil descriptivo de los acogimientos con familia extensa de la provincia de Málaga. Revista de Psicología Social Aplicada, 15(1-2), 93- 117.

McWey, L. y Mullis, K. A. (2004). Improving the lives of children in foster care: The impact of supervised visitation. Family Relations, 53(3), 293-300.

Molero, R. J., Moral, M. J., Albiñana, P., Sabater, Y. y Sospedra, R. (2007). Situación de los acogimientos en familia extensa en la ciudad de Valencia. Anales de Psicología, 23(2), 193200.

Montserrat, C. (2006). Acogimiento en familia extensa: un estudio desde la perspectiva de los acogedores, de los niños y niñas acogidos y de los profesionales que intervienen. Intervención Psicosocial, 15(2), 203-221.

Montserrat. C. (2007). Niños, niñas y adolescentes acogidos por sus familiares. ¿Qué sabemos, qué conocemos? Barcelona: Generalitat de Catalunya.

Oyserman, D. y Benbenishty, R. (1992). Keeping in touch:
Ecological factors related to foster care visitation. Child and Adolescent Social Work Journal, 9, 541-554.

Palacios, J. y Jiménez, J. (2007). Acogimiento familiar en Andalucía. Sevilla: Consejería para la Igualdad y Bienestar Social. Recuperado el 23 de septiembre de 2008 de: http://www.juntadeandalucia.es/observatoriodelainfancia/ OIA

Pecora, P. J., Le Prohn, N. S. y Nasuti, J. J. (1999). Role perceptions of kinship and other foster parents in family foster care. En R. Hegar y M. Scannapieco (Eds.), Kinship foster care: policy, practice, and research (pp. 155-178). New York: Oxford University Press.

Shlonsky, A., Webster, D. y Needell, B. (2003). The ties that bind: a cross-sectional analysis of siblings in foster care. Journal of Social Service Research, 29(3), 27-52.

Smith, M. C. (1996). An exploratory survey of foster mother and caseworker attitudes about sibling placement. Child Welfare, 75, 357-375.

Smith, M. C. (1998). Sibling placement in foster care: An exploration of associated concurrent preschool-aged child functioning. Children and Youth Services Review, 20, 389412.

Testa, M. F. y Slack, K. S. (2002). The gift of kinship foster care. Children and Youth Services Review, 24 (1-2), 79-108.

Fecha de recepción: 31 de marzo de 2009 Fecha de aceptación: 24 de abril de 2009

Notas del autor

El acceso a las familias y a la información sobre los acogimientos se realizó con la colaboración del Servicio de Protección de Menores de la Delegación Provincial de Málaga (Junta de Andalucía) y las ICIFs (Instituciones Colaboradoras de Integración Familiar), Hogar Abierto e Infania. 